\title{
Early predictors of mortality in parkinsonism and Parkinson disease
}

\author{
A population-based study
}

David Bäckström, MD, Gabriel Granåsen, MSc, Magdalena Eriksson Domellöf, PhD, Jan Linder, MD, PhD, Susanna Jakobson Mo, MD, PhD, Katrine Riklund, MD, PhD, Henrik Zetterberg, MD, PhD,

Kaj Blennow, MD, PhD, and Lars Forsgren, MD, PhD

Neurology ${ }^{\circledR}$ 2018;91:e2045-e2056. doi:10.1212/WNL.0000000000006576

\section{Abstract}

\section{Objective}

To examine mortality and associated risk factors, including possible effects of mild cognitive impairment, imaging, and CSF abnormalities, in a community-based population with incident parkinsonism and Parkinson disease.

\section{Methods}

One hundred eighty-two patients with new-onset, idiopathic parkinsonism were diagnosed from January 2004 through April 2009, in a catchment area of 142,000 inhabitants in Sweden. Patients were comprehensively investigated according to a multimodal research protocol and followed prospectively for up to 13.5 years. A total of 109 patients died. Mortality rates in the general Swedish population were used to calculate standardized mortality ratio and expected survival, and Cox proportional hazard models were used to investigate independent predictors of mortality.

\section{Results}

The standardized mortality ratio for all patients was 1.84 (95\% confidence interval 1.50-2.22, $p$ $<0.001$ ). Patients with atypical parkinsonism (multiple system atrophy or progressive supranuclear palsy) had the highest mortality. In early Parkinson disease, a mild cognitive impairment diagnosis, freezing of gait, hyposmia, reduced dopamine transporter activity in the caudate, and elevated leukocytes in the CSF were significantly associated with shorter survival.

\section{Conclusion}

Although patients presenting with idiopathic parkinsonism have reduced survival, the survival is highly dependent on the type and characteristics of the parkinsonian disorder. Patients with Parkinson disease presenting with normal cognitive function seem to have a largely normal life expectancy. The finding of a subtle CSF leukocytosis in patients with Parkinson disease with short survival may have clinical implications.

\author{
Correspondence \\ Dr. Bäckström \\ david.backstrom@umu.se
}

\section{RELATED ARTICLES}

\section{Editorial}

Life expectancy in

Parkinson disease

Page 991

\section{Patient Page}

Parkinson disease and mortality: Understanding how the two are connected Page e2106

\section{MORE ONLINE}

\section{ค Podcast}

Dr. Jeffery Ratliff interviews Dr. David Bäckström on a population-based study investigating early predictors of mortality in parkinsonism and Parkinson disease. NPub.org/patgbx

- CME Course

NPub.org/cmelist

From the Department of Pharmacology and Clinical Neuroscience (D.B., M.E.D., J.L., L.F.), Epidemiology and Global Health Unit, Department of Public Health and Clinical Medicine (G.G.), Department of Psychology (M.E.D.), and Department of Radiation Sciences, Diagnostic Radiology and Umeå Center for Functional Brain Imaging (S.J.M., K.R.), Umeå University; Institute of Neuroscience and Physiology (H.Z., K.B.), Department of Psychiatry and Neurochemistry, Sahlgrenska Academy at University of Gothenburg, Mölndal; Clinical Neurochemistry Laboratory (H.Z., K.B.), Sahlgrenska University Hospital, Mölndal, Sweden; Department of Molecular Neuroscience (H.Z.), University College London Institute of Neurology; and UK Dementia Research Institute at UCL (H.Z.), London, UK.

Go to Neurology.org/N for full disclosures. Funding information and disclosures deemed relevant by the authors, if any, are provided at the end of the article. 


\section{Glossary}

$\mathbf{A} \boldsymbol{\beta}_{42}=\beta$-amyloid 1-42; $\mathbf{C I}=$ confidence interval; DAT $=$ dopamine active transporter $\mathbf{M C I}=$ mild cognitive impairment; MMSE = Mini-Mental State Examination; MSA = multiple system atrophy; PD = Parkinson disease; PDD = Parkinson disease dementia; PIGD = postural imbalance and gait disorder; PSP = progressive supranuclear palsy; SMR = standardized mortality ratio; TUG = Timed Up and Go; UPDRS = Unified Parkinson’s Disease Rating Scale.

In Parkinson disease (PD), the second most common neurodegenerative disorder, life expectancy is reduced. ${ }^{1}$ There are, however, conflicting data regarding the size of, and specific factors accounting for the reduced survival in comparison to the general population. Most studies of survival in PD have been hospital-based or have used register-based case-finding methods. These designs may produce biased results, through underrepresentation of mild PD cases and lack of referral of older patients to hospital clinics. ${ }^{2}$ There are also few studies of the survival in unselected populations of patients with new-onset idiopathic parkinsonism (including atypical parkinsonism), rather than $\mathrm{PD}$.

In $\mathrm{PD}$, previous studies have found that a nontremordominant phenotype, PD dementia (PDD), and early autonomic dysfunction are associated with a shorter survival. ${ }^{1,3,4}$ The recently defined diagnosis of mild cognitive impairment in PD (PD-MCI $)^{5}$ has rarely been studied in this regard. Furthermore, the neurobiology of PD with short survival (in terms of factors such as striatal dopamine depletion patterns, CSF abnormalities, or APOE genotype) is not well known.

Against this background, we assessed all-cause mortality and associated risk factors in a population-based, Swedish cohort of patients with incident, carefully diagnosed idiopathic parkinsonism, including PD. The patients underwent extensive neurologic, neuropsychological, and laboratory testing as well as multimodal neuroimaging and received standard, or if indicated, advanced treatments by movement disorder neurologists during long-term follow-up.

\section{Methods}

\section{Study population}

All participants were part of a population-based incidence study of unselected cases of new-onset idiopathic parkinsonism, from a defined geographic catchment area of 142,000 inhabitants in northern Sweden. ${ }^{6}$ The local tradition is to refer all patients with suspected PD to the Department of Neurology at Umeå University Hospital (our department), which is the only neurologic department in the area. To avoid selection bias, and to make case identification as complete as possible, a population screening was performed through many sources, including letters sent twice yearly to all health practitioners asking for referral of all suspected cases with incident parkinsonism. Eldercare institutions were surveyed by visits (the largest institution) with an examination by neurologists of all cases that were reported to have signs of parkinsonism, or by interview (remaining institutions).

All patients were recruited to the study in the early motor (drug-naive) phase, between January 1, 2004, and April 30, 2009. After exclusion of patients with secondary parkinsonism (e.g., due to neuroleptic drugs or stroke) or dementia at baseline (e.g., patients with dementia with Lewy bodies), 182 participants were included and followed prospectively. A diagnosis of $\mathrm{PD}$, multiple system atrophy (MSA), or progressive supranuclear palsy (PSP) required agreement among the examiners (neurologists specialized in movement disorders) that the clinical criteria for the diagnosis were fulfilled based on the UK Parkinson's Disease Society Brain Bank criteria ${ }^{7}$ or criteria for MSA or PSP. ${ }^{8,9}$

At the latest follow-up (up to death or 8.5-13.5 years following inclusion in alive patients), 143 patients were diagnosed with PD, 13 with MSA, 18 with PSP, 4 as having unclassifiable parkinsonism, and 4 did not have idiopathic parkinsonism (figure 1). Autopsy confirmed the diagnosis in 3 cases of $\mathrm{PD}$ and 2 cases of PSP.

\section{Standard protocol approvals, registrations, and patient consents}

All participants provided written informed consent. The study was approved by the Regional Medical Ethics Board in Umeå, Sweden, and was performed in accord with the Declaration of Helsinki.

\section{Clinical assessments}

All participants were investigated at study entry (baseline) and followed up prospectively with standardized clinical examinations, including the Unified Parkinson's Disease Rating Scale (UPDRS), the modified Hoehn and Yahr Scale, Mini-Mental State Examination (MMSE), and the 39-item Parkinson's Disease Questionnaire, at least yearly, as described previously. ${ }^{10}$ We divided the baseline UPDRS scores into subscores for tremor (sum of items 20 and 21) and postural imbalance and gait disorder (PIGD; sum of items 13, $14,15,29$, and 30) and classified the phenotype of PD as tremor predominant, intermediate, or PIGD in accordance with the DATATOP (Deprenyl and Tocopherol Antioxidative Therapy of Parkinson's Disease) trial analyses. ${ }^{11}$ Olfactory function was investigated by the 12-item Brief Smell Identification Test, ${ }^{12}$ global mobility by the Timed Up and Go (TUG) test, which is the time it takes to rise up from a chair, walk $3 \mathrm{~m}$, and sit down again, ${ }^{13}$ and depression by the Montgomery-Åsberg Depression Rating Scale. ${ }^{14}$ Severe 


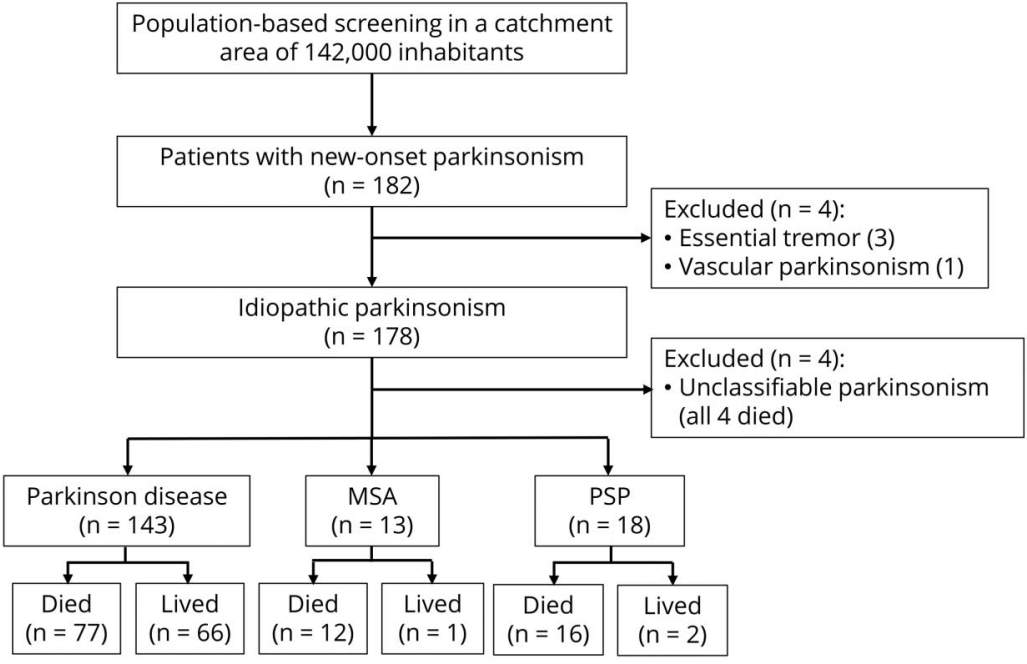

Diagnosis was established according to clinical diagnosis at the latest follow-up and confirmed by autopsy in 5 patients. MSA = multiple system atrophy; PSP = progressive supranuclear palsy. hyposmia is defined by a score $<4$ on the Brief Smell Identification Test. A possible REM sleep behavior disorder was defined by a history of dream enactment behavior on a screening questionnaire.

\section{CSF analysis}

At study entry, 99 of the patients with PD and 21 of the patients with atypical parkinsonism agreed to CSF collection by lumbar puncture, using standard procedures. CSF total tau concentration was measured using a sandwich ELISA (INNOTEST hTAU Ag; Fujirebio, Ghent, Belgium) constructed to determine all tau isoforms irrespective of phosphorylation status. ${ }^{15}$ Tau phosphorylated at threonine 181 was measured by a sandwich ELISA (INNOTEST PHOSPHO-TAU [181P]; Fujirebio). ${ }^{16}$ CSF $\beta$-amyloid 1-42 $\left(\mathrm{A} \beta_{42}\right)$ levels were measured using a sandwich ELISA (INNOTEST $\beta$-AMYLOID [1-42]; Fujirebio) constructed to measure $A \beta$ containing the first and 42 nd amino acids. ${ }^{17}$ CSF levels of $\alpha$-synuclein were analyzed using a commercially available $\alpha$-synuclein human ELISA (KHB0061; Invitrogen, Carlsbad, CA) according to instructions by the manufacturer. Experienced, board-certified laboratory technicians performed the CSF analyses using procedures approved by the Swedish Board for Accreditation and Conformity Assessment. Cells in the CSF were counted by automated flow cytometry. A subtle pleocytosis of mononuclear leukocytes was operationally defined by counts ranging between 2 and 20 cells per microliter. However, no patient had more than 10 cells per microliter.

\section{Dopamine active transporter imaging}

Of the 182 patients enrolled in the study, 170 patients (93.4\%) underwent dopamine active transporter (DAT) imaging by

${ }^{123}$ I-FP-CIT (DaTSCAN; GE Healthcare BV, Eindhoven, The Netherlands) SPECT. DAT imaging was done 3 hours following an IV bolus dose of $185 \mathrm{MBq}^{123} \mathrm{I}$-FP-CIT. Imaging was done prior to commencement of medication at baseline. The imaging protocol was done within the framework of a nonprofit clinical trial (EU no. 2009-011748-20) and constituted a substudy within the research project. Semiquantitative analysis (based on regions of interest) and visual evaluation of the DAT SPECT were done unbiased by any clinical information at all times. Normal reference values were derived from an age-matched group of healthy controls participating in the study, and reduction of DAT uptake in the patients with PD was measured in percent and SDs of the normal values. The most affected side (left or right) was defined by the putamen and caudate that showed the largest reduction of ${ }^{123}$ I-FP-CIT uptake. The putamen and caudate were investigated separately. The imaging protocol, equipment, and semiquantitative evaluation methods that were used have been described earlier. ${ }^{18}$ Two different SPECT cameras were used during the course of the project; one brain-dedicated SPECT camera (the Neurocam) was later substituted by a multipurpose hybrid SPECT/CT (both General Electric, Milwaukee, WI). Normal reference values were established for both equipments. ${ }^{18,19}$ All PD, MSA, and PSP patients fulfilling diagnostic criteria and who participated in the DAT imaging $(n=163)$ had a pathologic scan.

\section{Genetic testing}

One hundred thirty-three of the patients with $\mathrm{PD}$ agreed to DNA analysis by peripheral blood sampling. DNA was isolated from peripheral blood using standard procedures. The variations of interest (the $\varepsilon 2 / \varepsilon 3 / \varepsilon 4$ polymorphisms) in the APOE gene were genotyped using TaqMan Assays-by-Design (Applied Biosystems, Foster City, CA). The assay was performed according to manufacturer's instructions and analyzed using the allelic discrimination function of the TaqMan 7900 HT Fast Real-Time PCR system (Applied Biosystems). Genotype success rates of $100 \%$ were obtained. The patients who declined all laboratory investigations $(n=10)$ were older 
than the others (79.1 vs 70.5 years) and had slightly higher UPDRS scores for motor dysfunction but were otherwise comparable. All laboratory analyses were performed blinded from clinical data.

\section{Neuropsychology}

Extensive neuropsychological testing, used for PD-MCI and $\mathrm{PDD}^{20}$ diagnostics, was performed at baseline and after 1, 3, 5, and 8 years. At baseline, the patients with PD could be divided into patients with MCI (PD-MCI, $\mathrm{n}=61$ ) and patients without PD-MCI (PD with normal cognition, $\mathrm{n}=82$ ). Because all cognitive domains had been covered in the test battery throughout the study period, PD-MCI diagnoses were applied according to Movement Disorder Society guidelines, ${ }^{5}$ using level 2 criteria. ${ }^{21}$ Patients were classified as having MCI at baseline if (1) impaired in a minimum of 2 tests in one domain (single-domain MCI) or in a minimum of one test in 2 different domains (multiple-domain MCI), (2) impairments were $\geq 1.5$ SDs below mean of normative data, (3) selfperceived cognitive decline was reported in a questionnaire (e.g., the 39-item Parkinson's Disease Questionnaire) and/or directly by patient and/or family member, and (4) the patient had no functional impairment in basic activities of living (i.e., driving a car, social or personal care, medication management) due to cognitive impairment. The tests used for MCI classification were, for episodic memory: Free and Cued Selective Reminding Test, Logical Memory and Paired Associative Learning from the Wechsler Memory Scale, and Brief Visuospatial Memory Test (total recall); for working memory: Digit Span Forward and Digit Span Backward from Wechsler Adult Intelligence Scale III; for attention: Trail Making Test A and B; for language: Controlled Oral Word Association and Boston Naming Test; for visuospatial function: the Benton Judgment of Line Orientation Test and Pentagon Copying from MMSE; and for executive function: Wisconsin Card Sorting Test-computer version 2, Mental Control from Wechsler Memory Scale, and Category Fluency (animals in 60 seconds). In the multivariable analysis of factors predicting mortality in $\mathrm{PD}$, baseline neuropsychology results were used. However, to investigate possible effects of incident dementia (of which there were no cases at baseline), the neuropsychology results from the 3-year follow-up were also analyzed in relation to mortality. Incident PDD and PDMCI were diagnosed at the 3-year follow-up on the basis of previous test results, a documented decline, cognitive decline reported by the patient and/or family member, and (for PDD) by the occurrence of functional impairment in basic activities of living caused by cognitive impairment. Structural MRI and routine laboratory tests were performed to exclude cognitive impairment due to other causes than PD-MCI or PDD.

\section{Mortality}

We followed all surviving patients yearly for approximately 8.5 to 13.5 years, until August 31, 2017. The average time since inclusion in all patients was 7.7 years. Although a few older patients were followed by telephone, rather than visits, during the last few years, the survival data were complete. A death certificate, in which the cause of death was stated, was obtained for 98 (90\%) of all the fatalities in the cohort.

\section{Statistics}

Potential group differences in clinical variables at baseline were examined by 1-way analysis of variance, Fisher exact test, and Kruskal-Wallis test, and correlation between variables by Person $r$ and Spearman $\rho$, as appropriate. Post hoc contrasts in analysis of variance are shown corrected for multiple comparisons using Tukey HSD (honestly significant difference). The age- and sex-specific standardized mortality ratio (SMR) was calculated, stratified by clinical diagnosis and sex and, in the patients with PD, by MCI status, by dividing the observed number of deaths counted from baseline in each group by the expected number of deaths in each group. The expected numbers of deaths were calculated using the age- and sexspecific official Swedish National Statistics of 2004-2017 mortalities multiplied by the person-time from each group in the study. Confidence intervals (CIs) for the SMRs were calculated to the $95 \%$ level using the Poisson distribution and $p$ values using the $\chi^{2}$ distribution. Life expectancies (anticipated mean remaining time to live) based on SMR were calculated using a modified Gompertz function, as done previously. ${ }^{22}$ Cox proportional hazard analysis was used to investigate whether one or more covariates predicted mortality in PD. A list of potential risk factors for mortality was generated, including factors previously found predictive and factors of neurobiological interest (biomarkers). To investigate dopaminergic denervation at baseline, DAT-uptake ratios were normalized by average SDs above or below the normal mean (i.e., $z$ score) in order to equate the numerical values derived from the 2 different scanners that were used. The number of mononuclear leukocytes in CSF was investigated as a continuous predictor variable in relation to survival. The presence of a CSF leukocytosis was also investigated. For univariate comparisons, we controlled for a false discovery rate of 0.05 by using the Benjamini-Hochberg procedure, resulting in a significance level of $p<0.017$. Because age is strongly related to survival, all results were adjusted for age. Multivariable models were then developed using age with clinical variables and age with biomarker variables as predictors of survival. Variables significantly associated with survival at the $p<0.2$ level in the univariate models were included, using a backward elimination procedure. If variables were highly correlated, the variable with the lower $p$ value was included to avoid multicollinearity. The only exception was the PIGD score, which was correlated to TUG results but chosen over the TUG test because of perceived higher clinical usefulness. In the final multivariable models, only variables with $p<0.005$ were included to avoid overfitting of the model and to restrict the number of predictors for ease of use in clinical practice. To avoid confounding related to the heterogeneity of the different parkinsonian diseases, detailed prognostic models were developed only for $\mathrm{PD}$, except for the CSF protein markers, which were also investigated in atypical parkinsonism. All statistical analyses 
were performed using SPSS 23.0 (IBM Corp., Armonk, NY) or R Statistical Software.

\section{Data availability}

Anonymized data can be obtained by request from any qualified investigator for purposes of replicating procedures and results.

\section{Results}

\section{Survival in incident idiopathic parkinsonism}

Clinical characteristics at baseline for the patients with idiopathic parkinsonism are shown in table 1 . Survival data from first evaluation to death or end of the study were obtained for all participants (figure 1). Of the 178 patients with idiopathic parkinsonism, 109 (61.2\%) died during follow-up. Seventyseven (53.8\% of 143$)$ of the deaths occurred in the PD group, 12 (92.3\% of 13$)$ in the MSA group, and 16 (88.9\% of 18$)$ in the PSP group. The 4 patients with unclassifiable parkinsonism likely represent cases of late-onset PD but were excluded from further analyses, as they did not fulfill specific diagnostic criteria. The overall mean age at death was 82.0 years. Deep brain stimulation or pumps for intestinal delivery of levodopa were used or had been used by $12(8.4 \%)$ of the 143 patients with PD.

Survival was related to the age at the first visit (hazard ratio for death: 3.03 for each decade older, $95 \%$ CI $2.30-3.98, p<$ $0.001)$. However, when survival in patients with MSA or PSP was analyzed separately from $\mathrm{PD}$, the age at the first visit was not a significant factor $(p=0.766)$. As a measurement of global cognitive function, survival also correlated with the baseline MMSE score after adjustment for age (1.19 times higher hazard for death for each lower point, $p=0.006)$. The SMR for the whole parkinsonism cohort was $1.84(p<0.001)$ times higher than the comparable age and sex distributionstandardized mortality of the Swedish population during the years 2004 to 2017 (table 2).

The SMR for the patients with PD was 1.58 and 3.32 for the patients with atypical parkinsonism (table 2). The patients with PD had an age-adjusted hazard ratio for death of 0.43 (95\% CI 0.27-0.67, $p<0.001$ ) compared to the patients with atypical parkinsonism. More specifically, the age-adjusted hazard ratio for death in MSA was 2.76 (95\% CI 1.48-5.15, $p=0.001)$ and $1.42(95 \%$ CI $1.08-1.88, p=0.012)$ in PSP compared to the patients with PD (figure 2). As shown

Table 1 Characteristics of participants at the first (baseline) visit

\begin{tabular}{|c|c|c|c|c|c|}
\hline Variable & $\begin{array}{l}\text { Parkinson } \\
\text { disease }\end{array}$ & MSA & PSP & $\begin{array}{l}\text { Unclassified } \\
\text { parkinsonism }\end{array}$ & $p$ Value \\
\hline No. of participants & 143 & 13 & 18 & 4 & \\
\hline Age, y & $71.2(9.8)$ & $73.6(9.6)$ & $75.0(7.1)$ & $85.7(7.5)$ & $0.012^{\mathrm{b}}$ \\
\hline Disease duration, y & $1.8(1.4)$ & $1.9(0.9)$ & $2.1(1.5)$ & $2.6(1.0)$ & 0.670 \\
\hline Sex, M/F (\% M) & $85 / 58(59)$ & $8 / 5(61)$ & $8 / 10(44)$ & $2 / 2(50)$ & 0.648 \\
\hline Hoehn and Yahr, median (IQR) & $2.0(2.0-3.0)$ & $2.5(2.0-3.0)$ & $2.5(2.0-3.0)$ & $3.0(2.5-3.5)$ & 0.009 \\
\hline UPDRS total, median (IQR) & $35.0(27.0-46.0)$ & $37.0(32.0-49.0)$ & $35.0(31.0-45.0)$ & $50.5(41.5-64.5)$ & 0.283 \\
\hline UPDRS III, median (IQR) & $26.0(19.0-35.0)$ & $23.0(20.0-37.0)$ & $26.5(24.0-32.0)$ & $35.0(28.0-41.5)$ & 0.541 \\
\hline Possible RBD, n (\%) & $15(11)$ & $2(15)$ & $0(0)$ & $0(0)$ & 0.286 \\
\hline MADRS, depression score & $4.5(3.7)$ & $8.0(7.4)$ & $7.7(9.4)$ & $5.0(0.0)$ & 0.025 \\
\hline Smell test, no. correct & $6.6(2.7)$ & $7.0(3.3)$ & $7.1(2.3)$ & & 0.626 \\
\hline Freezing, ever, n (\%) & $95(67)$ & $9(69)$ & $13(76)$ & $4(100)$ & 0.508 \\
\hline Orthostatic blood pressure drop, ${ }^{a} \mathrm{~mm} \mathrm{Hg}$ & $10.9(18.8)$ & $21.3(22.2)$ & $2.7(15.0)$ & & $0.048^{c}$ \\
\hline MMSE score & $28.5(1.7)$ & $29.0(1.1)$ & $27.9(1.6)$ & $27.3(2.1)$ & 0.181 \\
\hline $\mathrm{MCl}$ diagnosis, $\mathrm{n}(\%)$ & $61(43)$ & $3(23)$ & $14(78)$ & $3(75)$ & $0.007^{d}$ \\
\hline
\end{tabular}

Abbreviations: IQR = interquartile range; MADRS = Montgomery-Åsberg Depression Rating Scale; $\mathrm{MCI}=$ mild cognitive impairment; MMSE = Mini-Mental State Examination; MSA = multiple system atrophy; PSP = progressive supranuclear palsy; RBD = REM sleep behavior disorder; UPDRS = Unified Parkinson's Disease Rating Scale, Part III, is the motor function subscale.

The participants were naive to dopaminergic treatment at this time. Values are means (SD) unless otherwise stated. Disease duration is the time from onset of first motor symptom, as recalled by the patient, to baseline.

a Systolic pressure difference after 3 minutes of standing. Post hoc comparisons, using Tukey honestly significant difference with the significance level of $p<$ 0.05 to control for multiple testing, showed the following differences.

${ }^{b}$ Parkinson disease younger than unclassified parkinsonism.

${ }^{c}$ MSA more orthostatic than PSP.

${ }^{\mathrm{d}} \mathrm{MCI}$ more common in PSP than in Parkinson disease and than in MSA. 
Table 2 Standardized mortality ratio in parkinsonism in relation to clinical diagnosis

\begin{tabular}{|c|c|c|c|c|c|}
\hline Sex & No. & No. of events & Expected & SMR $(95 \% \mathrm{CI})$ & $p$ Value \\
\hline \multicolumn{6}{|c|}{ Idiopathic parkinsonism cohort, all cases } \\
\hline Female & 73 & 48 & 21.3 & $2.25(1.66-2.98)$ & $<0.001$ \\
\hline Male & 101 & 57 & 35.8 & $1.59(1.21-2.06)$ & $<0.001$ \\
\hline Total & 174 & 105 & 57.1 & $1.84(1.50-2.22)$ & $<0.001$ \\
\hline \multicolumn{6}{|c|}{ Atypical parkinsonism } \\
\hline Total & 31 & 28 & 8.4 & $3.32(2.21-4.80)$ & $<0.001$ \\
\hline \multicolumn{6}{|c|}{ Parkinson disease } \\
\hline Female & 58 & 34 & 16.3 & $2.09(1.45-2.91)$ & $<0.001$ \\
\hline Male & 85 & 43 & 32.4 & $1.33(0.96-1.79)$ & 0.077 \\
\hline Total & 143 & 77 & 48.7 & $1.58(1.25-1.98)$ & $<0.001$ \\
\hline \multicolumn{6}{|c|}{ Parkinson disease with normal cognition } \\
\hline Female & 37 & 17 & 10.8 & $1.57(0.91-2.51)$ & 0.085 \\
\hline Male & 45 & 18 & 18.5 & $0.97(0.58-1.54)$ & 0.814 \\
\hline Total & 82 & 35 & 29.3 & $1.19(0.83-1.66)$ & 0.341 \\
\hline \multicolumn{6}{|c|}{ Parkinson disease with $\mathrm{MCl}$} \\
\hline Female & 21 & 17 & 5.5 & $3.12(1.82-4.99)$ & $<0.001$ \\
\hline Male & 40 & 25 & 13.9 & $1.80(1.162-2.65)$ & 0.005 \\
\hline Total & 61 & 42 & 19.4 & $2.17(1.56-2.93)$ & $<0.001$ \\
\hline
\end{tabular}

Abbreviations: $\mathrm{Cl}$ = confidence interval; $\mathrm{MCl}$ = mild cognitive impairment; $\mathrm{SMR}=$ standardized mortality ratio.

in table 3, the most common cause of death was pneumonia, but in many cases, the exact cause of death could not be determined (e.g., patients who died alone in their homes). Assuming the mean age of 71.7 years at baseline in idiopathic parkinsonism, the expected survival in PD was 9.6 years, and 6.1 years in atypical parkinsonism.

\section{Survival in PD}

Although there was no sex difference in the hazard ratio for death in the patients with PD, the SMR was higher in female (2.09, i.e., $109 \%$ more cases of death in female patients with PD than in the general female Swedish population) than in male (SMR: 1.33) patients (table 2). The survival also correlated with cognitive status. Mortality in patients with PD (both male and female) with normal cognition at baseline was not significantly different from the general Swedish population, while it was increased in patients with PD-MCI (SMR: 2.17; table 2). The patients with PD-MCI at baseline had a 2.4 times higher age-adjusted hazard of death during follow-up compared to the patients without $\mathrm{MCI}$ at baseline $(p<0.001$, table 4$)$. Survival in PD also correlated with the baseline MMSE score (1.17 times higher hazard for death for each lower point, after adjustment for age, $p=0.011$ ). Fewer years of formal education was not associated with reduced survival. Assuming the mean age of 71.2 years at baseline, the expected survival in PD was 11.6 years without $\mathrm{MCI}$ and 8.2 years with MCI.

A baseline PD phenotype comprising MCI, freezing of gait, a PIGD phenotype, hyposmia, high disease severity (measured by the total and Part III UPDRS scores), slowness in the TUG test, and onset of dementia during the first 3 years predicted shorter survival (table 4 and figure $3, \mathrm{~A}-\mathrm{F}$ ) in the univariate analysis. Tremor was uncorrelated to survival. The patients with PD had a 38\% lower DAT uptake in the putamen and a $24 \%$ lower DAT uptake in the caudate nucleus in the most affected hemisphere compared to healthy controls. A neurobiological factor (biomarker) that predicted a shorter survival in PD was reduced DAT uptake in the striatum, particularly in the caudate nucleus (1.48 times higher hazard for death for each SD of lower uptake), while the baseline CSF protein concentrations of neurodegenerative markers and APOE genotype were not predictive (table 4). Higher $A \beta_{42}$ concentration in CSF was, however, moderately associated with increased survival when the whole cohort of patients with parkinsonian disorders was investigated (age-adjusted hazard ratio: 0.99 per $\mathrm{pg} / \mathrm{mL}, 95 \% \mathrm{CI} 0.98-1.00, p=0.048$ in all patients and $0.97 \mathrm{per} \mathrm{pg} / \mathrm{mL}, 95 \%$ CI $0.95-1.00, p=0.036$ in the patients with atypical parkinsonism). Furthermore, a proportion of all patients with PD (13.1\%) had a subtle 


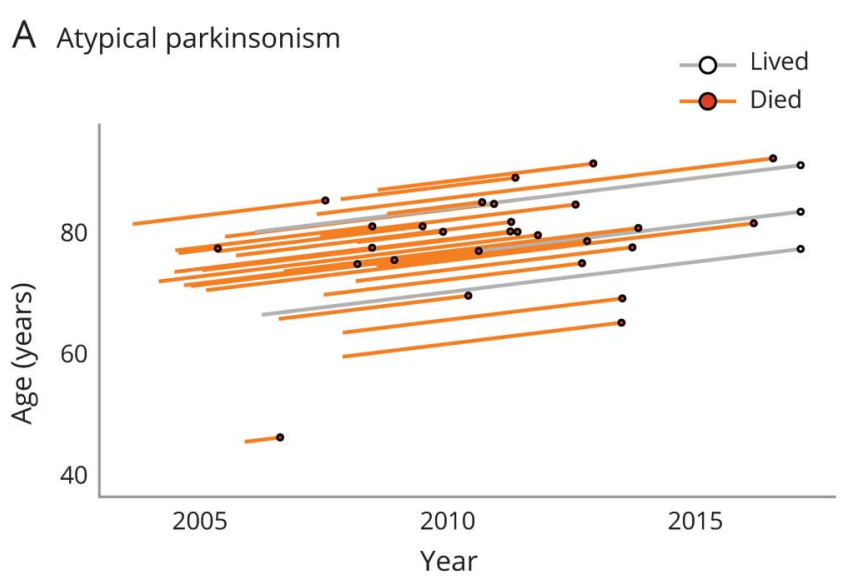

Parkinson disease

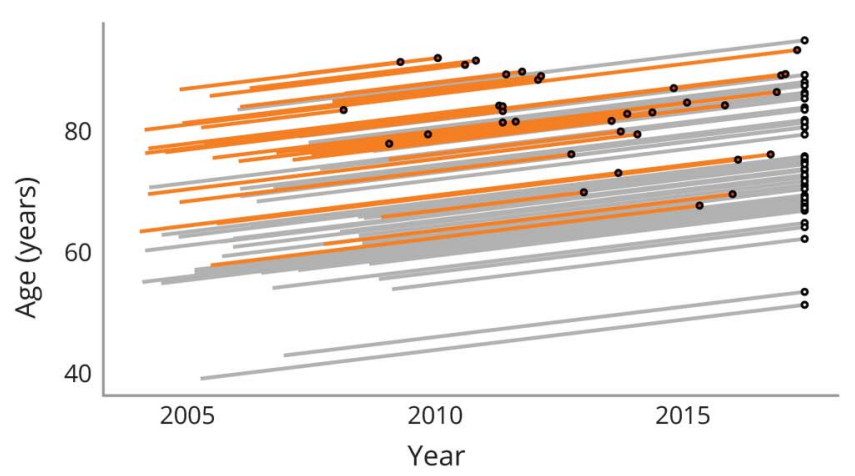

B

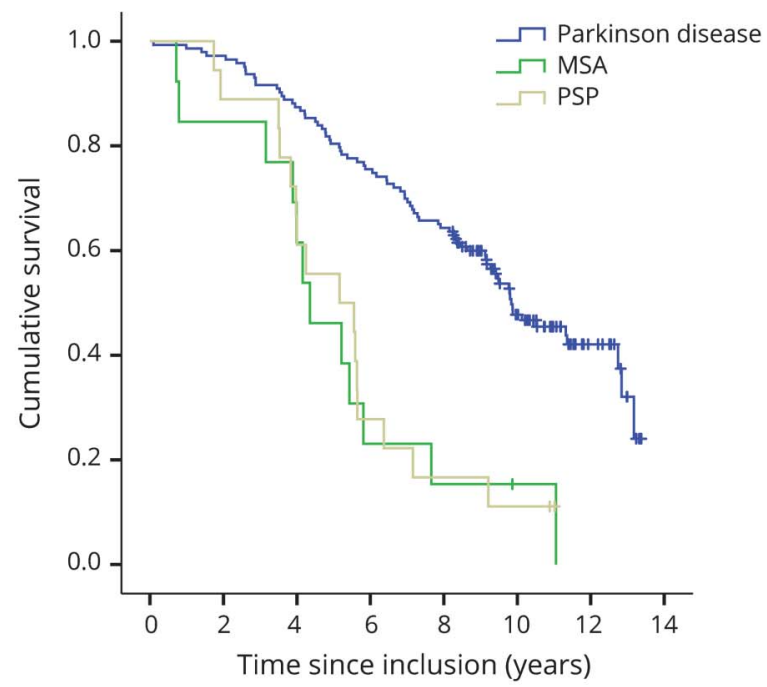

(A) Lexis diagram showing follow-up of patients throughout the study. The "atypical parkinsonism" group comprises patients with new-onset MSA and PSP. (B) Kaplan-Meier plot of survival in relation to diagnosis (for number at risk, see supplemental table e-1, links.Iww.com/WNL/A762). MSA = multiple system atrophy; PSP = progressive supranuclear palsy.

pleocytosis of mononuclear leukocytes (2-10 cells) in the CSF. These patients had a significantly worse prognosis compared to the other patients (hazard ratio for death: 6.31, $p<0.001$; table 4), independently of adjustment for erythrocytes. Given this finding, the CSF results were further analyzed. There was a trend that a higher number of leukocytes at baseline tended to correlate with higher CSF total tau and phosphorylated tau concentrations $(r=0.24, p=$ 0.019 and $r=0.22, p=0.028$, respectively). There was no evidence of CNS infection in any of the patients and there was no difference in neurodegenerative marker or total protein concentrations in the CSF between PD patients with a leukocytosis and the other PD patients. However, the PD patients with a CSF leukocytosis had a higher progression of the UPDRS III scores at the 1-year follow-up (median 4-point increase vs 3-point decrease, $p=0.007$, Mann-Whitney $U$ test) than the other patients with $\mathrm{PD}$, despite using equal medication dosages (as measured by levodopa-equivalent daily dose).

After excluding factors strongly correlated to each other and including only strictly significant factors (at the $p<0.005$ level) in a multivariable model, older age at baseline, prevalent mild cognitive impairment, higher PIGD score, hyposmia, reduced DAT uptake in the caudate, and an inflammatory reaction (leukocytosis) in the CSF were independent clinical and biomarker predictors of reduced survival in PD.

\section{Discussion}

In this prospective, population-based cohort study involving in-person examination of all participants, we evaluated the prognosis of idiopathic parkinsonism and $\mathrm{PD}$ with respect to mortality, stratified by factors related to MCI and clinical and neurobiological characteristics. To avoid selection bias, we included all patients with incident idiopathic parkinsonism in the studied area, rather than only PD, and we explicitly diagnosed atypical forms of parkinsonism. Our results should, therefore, provide information that is generalizable to the "real-life" experience of the overall population with idiopathic parkinsonism.

In line with the findings from several previous studies, ${ }^{23,24}$ patients with MSA and PSP had a markedly worse prognosis than patients with PD. In the present cohort, the patients with incident, atypical parkinsonism (i.e., MSA or PSP) were older than in most previous, hospital-based studies, ${ }^{24-26}$ possibly 
Table 3 Causes of death in parkinsonism in relation to clinical diagnosis

\begin{tabular}{|c|c|c|c|c|}
\hline Variable & PD & MSA & PSP & $p$ Value \\
\hline Deaths/no. of participants & $77 / 143$ & $12 / 13$ & $16 / 18$ & $<0.001$ \\
\hline Mean age at death (SD) & $83.0(6.3)$ & $78.3(11.0)$ & $80.0(7.1)$ & 0.073 \\
\hline Mean survival, years from baseline to death (SD) & $6.5(3.0)$ & $4.7(2.8)$ & $4.8(1.9)$ & 0.031 \\
\hline Pneumonia & $15(19.5)$ & $3(25.0)$ & $7(43.8)$ & 0.116 \\
\hline Dementia & $2(15.6)$ & 0 & 0 & 0.085 \\
\hline Unknown & $9(11.7)$ & 0 & $2(12.5)$ & 0.451 \\
\hline Heart infarction & $7(9.1)$ & $1(8.3)$ & $1(6.3)$ & 0.934 \\
\hline Cancer, all types & $6(7.8)$ & $2(16.7)$ & $1(6.3)$ & 0.556 \\
\hline Heart failure & $4(5.2)$ & $2(16.7)$ & 0 & 0.159 \\
\hline Other causes & $24(31.2)$ & $4(33.3)$ & $5(31.3)$ & 0.989 \\
\hline
\end{tabular}

Abbreviations: $\mathrm{MSA}=$ multiple system atrophy; $\mathrm{PD}=$ Parkinson disease; $\mathrm{PSP}=$ progressive supranuclear palsy.

Data are $n(\%)$ unless otherwise stated. In 2 further patients with PD, pneumonia was determined to have contributed to death in combination with sepsis and renal failure, respectively. Difference in cause of death was calculated by $\chi^{2}$ analysis.

because of the population-based design. Our results confirm the dire prognosis of these diseases in this cohort, with a relatively high observed age at onset. In fact, age was not a significant predictor of survival in patients with MSA or PSP, which is likely explained by the strong effect on mortality caused by these disorders themselves.

Recent studies have also, despite advances in modern treatment, found a reduced life expectancy in PD. ${ }^{1}$ Similarly, we found that the survival of patients with PD was reduced in comparison with the general Swedish population during the same years (SMR 1.58, $p<0.001$ ). The SMR in most modern PD mortality studies has been in the range of 1.5 to $2.7 .^{1,2}$ Hence, the risk of death in the present study was in the lower range. This may be a result of the population-based study protocol (including patients with mild parkinsonism and explicitly diagnosing cases of atypical parkinsonism, that have a worse prognosis) and the fact that all patients had access to comprehensive health care services throughout the disease course.

It is currently unclear why many patients with PD experience shorter lifespans. In the present PD cohort, the excess mortality occurred most clearly in female patients and in patients with MCI (with pneumonia being the most common cause of death). Data on the general Swedish population show longer survival in women than in men. Therefore, a similar death rate (i.e., a hazard ratio close to 1) between the sexes in the present study, as well as in previous studies, ${ }^{27,28}$ may be interpreted to indicate a worse than expected prognosis for female patients with PD. In keeping with this, some previous PD studies have explicitly reported a higher mortality among female patients. ${ }^{29-31}$

The fact that life expectancy is reduced in PD compared to the general population independently from comorbidities, ${ }^{32}$ and the finding of a correlation between mortality and severity of
PD symptoms as measured by clinical scales, ${ }^{33,34}$ suggests that disease-specific features (such as $\alpha$-synuclein pathology) at least partly account for the increased mortality. We found that the increased mortality in PD correlated with core parkinsonian symptoms (with the notable exception of tremor) and olfactory dysfunction, independently of age. The elevated mortality in PD also correlated with the severity of striatal DAT imaging deficits, both in the putamen and in the caudate. Our findings indicate that the neurodegenerative process in $\mathrm{PD}$, which is linked to nigrostriatal denervation, is in itself associated with increased mortality. Of note, resting tremor in PD has been found to only weakly correlate with striatal dopaminergic denervation. ${ }^{35}$

In contrast, mortality was not increased in patients with $\mathrm{PD}$ who did not have cognitive impairment at study entry (male patients without MCI actually had an SMR below 1). The findings underscore the importance of an early PD-MCI diagnosis, as defined by the Movement Disorders Society. There is evidence that PD is associated with lower risk of some diseases, such as many cancers, and a lower risk factor burden from tobacco smoking and arterial hypertension. ${ }^{36-39}$ In $\mathrm{PD}$ patients with normal cognition, who have a milder disease phenotype, such differences could counterbalance mortality increases caused by neurodegeneration. Furthermore, a lower level of education was not associated with increased mortality. This may indicate that the higher mortality in patients with poorer cognitive function was related to the pathologic process leading to cognitive dysfunction, rather than being explained by socioeconomic factors. In keeping with this interpretation, a few previous studies have found dementia in PD to be an independent risk factor for death. ${ }^{3,40}$ Of note, we found higher mortality in PD patients with more severe caudal DAT-uptake deficits. Imaging studies (including an fMRI study of the same patients with PD as those in the 
Table 4 Early predictors of mortality in Parkinson disease

\begin{tabular}{|c|c|c|c|c|c|}
\hline \multirow[b]{2}{*}{ Variable, at baseline } & \multirow[b]{2}{*}{ Observed range } & \multicolumn{2}{|c|}{ Adjusted only for age } & \multicolumn{2}{|c|}{ Multivariable clinical model } \\
\hline & & HR $(95 \% \mathrm{Cl})$ & $p$ Value & HR $(95 \% \mathrm{Cl})$ & $p$ Value \\
\hline Age & \multicolumn{3}{|l|}{39.7 to $90.0 \mathrm{y}$} & $1.11(1.07-1.16)$ & $<0.001$ \\
\hline Duration, since first symptom & 0.2 to $7.5 \mathrm{y}$ & $0.93(0.81-1.08)$ & 0.366 & - & - \\
\hline Sex & Fvs $M$ & $1.03(0.65-1.63)$ & 0.909 & - & - \\
\hline UPDRS total score & 8 to 81 & $1.03(1.01-1.05)$ & $0.001^{c}$ & - & - \\
\hline UPDRS III subscore & 5 to 62 & $1.03(1.01-1.05)$ & $0.003^{c}$ & - & - \\
\hline PIGD score & 0 to 12 & $2.61(1.64-4.17)$ & $<0.001^{\mathrm{c}}$ & $3.25(1.75-6.05)$ & $<0.001$ \\
\hline Tremor score & 0 to 12 & $0.66(0.32-1.35)$ & 0.255 & - & - \\
\hline Freezing $^{a}$ & 0 to 3 & $2.17(1.40-3.36)$ & $<0.001^{\mathrm{c}}$ & - & - \\
\hline Falling ${ }^{a}$ & 0 to 2 & $0.90(0.45-1.79)$ & 0.753 & - & - \\
\hline Dysphagia $^{a}$ & 0 to 2 & $1.80(1.02-3.21)$ & 0.044 & - & - \\
\hline Body mass index & 19.2 to $36.5 \mathrm{~kg} / \mathrm{m}^{2}$ & $1.01(0.94-1.09)$ & 0.800 & - & - \\
\hline $\begin{array}{l}\text { Severity of hyposmia (correct } \\
\text { answers on B-SIT) }\end{array}$ & 0 to 12 & $0.83(0.75-0.92)$ & $<0.001^{c}$ & $0.84(0.75-0.93)$ & $<0.001$ \\
\hline Timed Up and Go test & 4.0 to $41.0 \mathrm{~s}$ & $1.11(1.07-1.15)$ & $<0.001^{c}$ & - & - \\
\hline Previous smoker & ever vs never & $1.54(0.94-2.51)$ & 0.081 & - & - \\
\hline MADRS, depression score & 0 to 18 & $1.02(0.95-1.09)$ & 0.529 & - & - \\
\hline Years of education & 6 to 30 & $1.01(0.93-1.10)$ & 0.780 & - & - \\
\hline Mild cognitive impairment & yes/no & $2.38(1.47-3.84)$ & $<0.001^{c}$ & $1.81(1.04-3.14)$ & 0.035 \\
\hline Dementia within first $3 y$ & yes/no & $1.94(1.12-3.34)$ & $0.017^{\mathrm{c}}$ & - & - \\
\hline Orthostatic blood pressure drop & -26 to $77 \mathrm{~mm} \mathrm{Hg}$ & $1.01(0.99-1.02)$ & 0.425 & - & - \\
\hline \multicolumn{4}{|l|}{ Biomarkers } & \multicolumn{2}{|l|}{$\begin{array}{l}\text { Biomarker } \\
\text { model }\end{array}$} \\
\hline Age & \multicolumn{3}{|l|}{43.6 to 86.8 y } & $1.17(1.11-1.23)$ & $<0.001$ \\
\hline APOE \&4 genotype & 0 to $2 \varepsilon 4$ alleles & $1.14(0.71-1.83)$ & 0.593 & - & - \\
\hline $\operatorname{CSF~A} \beta_{42}, p g / m L$ & 249 to $1,373 \mathrm{ng} / \mathrm{L}$ & $0.99(0.98-1.01)$ & 0.377 & - & - \\
\hline CSF t-tau, pg/mL & 81 to $1,053 \mathrm{ng} / \mathrm{L}$ & $1.01(0.99-1.02)$ & 0.240 & - & - \\
\hline CSF p-tau, pg/mL & 17 to $129 \mathrm{ng} / \mathrm{L}$ & $1.08(0.95-1.23)$ & 0.255 & - & - \\
\hline CSF a-synuclein, pg/mL & 323 to $2,179 \mathrm{ng} / \mathrm{L}$ & $1.02(0.95-1.09)$ & 0.594 & - & - \\
\hline Inflammatory reaction in CSF & yes/no & $\begin{array}{l}6.31 \\
(3.04-13.12)\end{array}$ & $<0.001^{\mathrm{c}}$ & $5.59(2.67-11.71)$ & $<0.001$ \\
\hline CSF leukocytes, mononuclear cells & 0 to 10 (count/ $\mu \mathrm{L}$ ) & $1.28(1.12-1.46)$ & $<0.001^{c}$ & - & - \\
\hline DAT uptake, most affected putamen & -5.16 to -0.38 & $0.60(0.44-0.82)$ & $0.002^{c}$ & - & - \\
\hline DAT uptake, most affected caudate & -4.72 to 0.14 & $0.67(0.53-0.85)$ & $0.001^{c}$ & $0.81(0.61-1.06)$ & 0.132 \\
\hline \multicolumn{6}{|c|}{$\begin{array}{l}\text { Abbreviations: } A \beta_{42}=\beta \text {-amyloid 1-42; B-SIT = Brief Smell Identification Test; } \mathrm{Cl}=\text { confidence interval; DAT = dopamine active transporter; HR = hazard ratio } \\
\text { MADRS = Montgomery-Ásberg Depression Rating Scale; PIGD = postural imbalance and gait difficulty; } \mathrm{p} \text {-tau = phosphorylated tau; t-tau = total tau; UPDRS } \\
\text { Unified Parkinson's Disease Rating Scale. } \\
\text { HRs for mortality in patients with Parkinson disease }(\mathrm{n}=143) \text {, within } 8.5 \text { to } 13.5 \text { years. The middle } 2 \text { columns show univariate association but, for clarity, values } \\
\text { are shown after adjustment for age. } \\
\text { a Assessed by UPDRS. } \\
\text { b The systolic pressure difference after } 3 \text { minutes of standing. Inflammatory reaction in CSF is defined by a subtle pleocytosis }(2-10 \text { mononuclear cells/ } \mu \mathrm{L}) \\
\text { 'Significant prior to inclusion in the multivariable model. }\end{array}$} \\
\hline
\end{tabular}


Figure 3 Survival in Parkinson disease in relation to phenotype
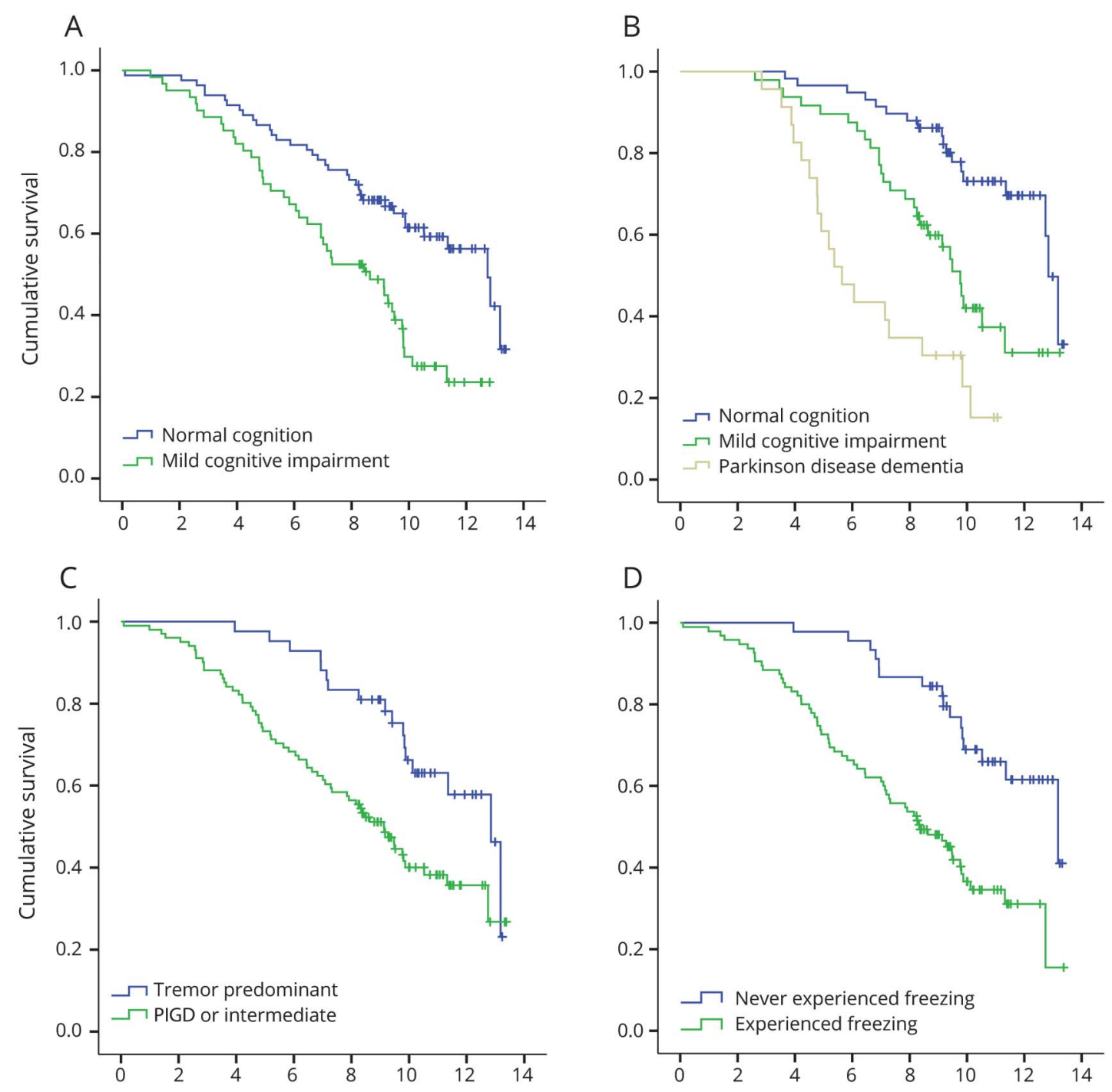

$\mathrm{D}$
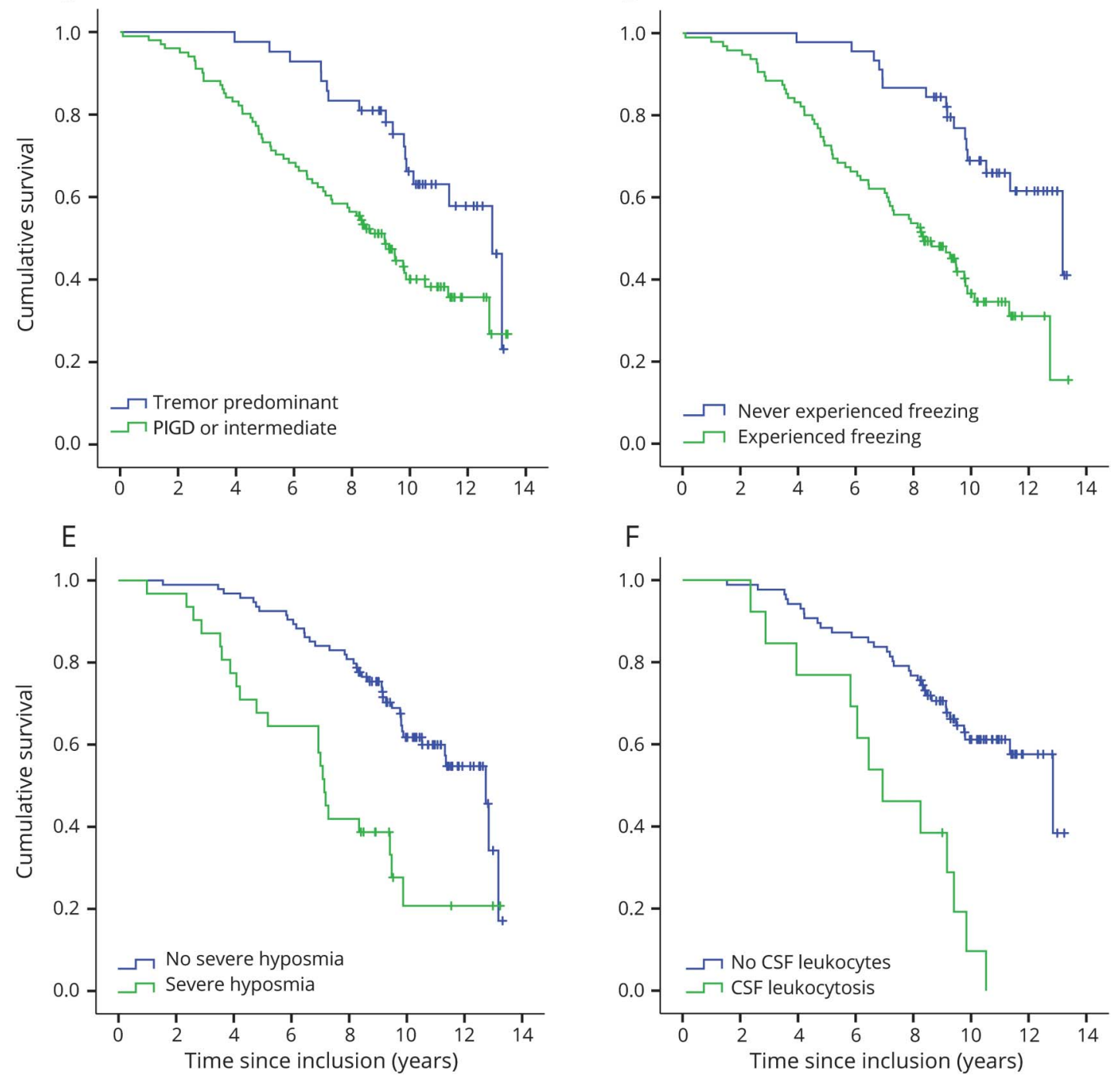

Kaplan-Meier plots of survival in patients with Parkinson disease $(n=143)$ in relation to clinical and neurobiological phenotype at baseline (except panel $B$ which is related to phenotype at 3 years). Severe hyposmia is defined by a B-SIT score $<4$. All variables (A-F) were significantly related to survival at the $p<$ 0.001 level (log rank) except the tremor or PIGD/intermediate variable (C), which was significant at the $p=0.004$ level (for number at risk, see supplemental table e-2, links.Iww.com/WNL/A762). B-SIT = Brief Smell Identification Test; PIGD = postural imbalance and gait disorder. 
present study) have shown that dopamine denervation in the caudate nucleus in PD correlates with cognitive deficits. ${ }^{41,42}$

A positive $A P O E \varepsilon 4$ carrier status or $\alpha$-synuclein, $A \beta_{42}$, and total tau or phosphorylated tau concentrations in CSF were uncorrelated with PD mortality. However, the number of CSF samples may have made our study underpowered for detecting such differences. The difference compared to the findings in a recent neuropathologic study ${ }^{43}$ could also relate to the fact that the present investigation used a more homogeneous disease group (PD). When CSF samples from all patients with idiopathic parkinsonism were included, a lower $A \beta_{42}$ concentration was associated with shorter survival. This may indicate that Alzheimer disease-type pathology is a predictor of reduced survival in parkinsonian disorders in general, ${ }^{43}$ mainly in MSA and/or PSP.

The finding of a low-grade inflammatory reaction in the CSF of $13.1 \%$ of the patients with PD was strongly related to a reduced survival (with a 6.31 times increased hazard for death). An increase of proinflammatory cytokines in the CNS and an inflammatory, hyperreactive state in circulating monocytes has previously been shown in PD. ${ }^{44,45}$ Two large, observational studies have also found a lower risk of PD associated with the use of nonsteroidal anti-inflammatory drugs in the general population. ${ }^{46,47}$ Taken together, our findings might suggest a triggered immune system, responding to the presence of abnormal, misfolded proteins in PD patients with short lifespans, possibly contributing to disease progression. The shorter lifespans in patients with a CSF leukocytosis indicate a rationale for further investigating immunomodulation to reduce PD mortality.

The possibility of uncontrolled confounding factors and the fact that neuropathologic diagnosis at autopsy of the nervous system was obtained in only 5 of 109 deaths are limitations of the study. Several studies have reported that neuropathologic confirmation of a clinical diagnosis of idiopathic PD ranges from $65 \%$ to $93 \%,{ }^{48}$ although the accuracy is higher in expert centers. ${ }^{49}$ In addition, no autopsy examinations were performed in any of the MSA cases. While acknowledging the limitations of a clinical diagnosis, the risk of incorrect diagnosis was nonetheless minimized by the long follow-up periods and the finding of pathologic uptake on DAT imaging in all of the examined patients. Even so, the results, especially the CSF findings, need to be confirmed by future studies. Our study also has several strengths, including a population-based design, a high proportion of patients who were examined using a multimodal research protocol, and prospective followup for up to 13.5 years.

The present study shows that patients with incident parkinsonism have reduced survival but that the survival is highly dependent on the type and characteristics of the parkinsonian disorder. Early MCI in PD is an important predictor of the prognosis. The finding of a low-grade immune reaction in the CSF of patients with PD who have short survival may have important clinical implications and therefore merits further investigation.

\section{Author contributions}

D.B., J.L., and L.F. designed the study. D.B. and G.G. conducted the statistical analysis with the help of L.F. D.B. wrote the first version of the report and contributed to the data collection. S.J.M and K.R. contributed to the data collection, statistical analysis, and writing of the report. M.E.D., J.L., H.Z., and K.B. contributed to the data collection and writing of the report.

\section{Acknowledgment}

Mona Edström, RN, and Jörgen Andersson, laboratory technician (Department of Pharmacology and Clinical Neuroscience, Umeå University), provided valuable assistance. The authors are grateful to all patients for their participation.

\section{Study funding}

The study was supported by grants from the Swedish Medical Research Council, Erling-Persson Foundation, Umeå University, Västerbotten County Council, King Gustaf V and Queen Victoria Freemason Foundation, Swedish Parkinson Foundation, Kempe Foundation, Swedish Parkinson's Disease Association, the Torsten Söderberg Foundation, the Swedish Brain Foundation, the European Research Council, and the Knut and Alice Wallenberg Foundation.

\section{Disclosure}

D. Bäckström, G. Granåsen, and M. Domellöf report no disclosures relevant to the manuscript. J. Linder reports receiving honoraria for lectures from GSK, Lundbeck, Boehringer Ingelheim, Abbott, AbbVie, Solvay, Orion Pharma, UCB, Nordic InfuCare, Medtronic, and IPSEN, and serving on advisory boards for Boehringer Ingelheim, Lundbeck, and GSK. S. Jakobson Mo and K. Riklund report no disclosures relevant to the manuscript. H. Zetterberg has served at advisory boards for Roche Diagnostics and Eli Lilly, has received travel support from Teva and is a cofounder of Brain Biomarker Solutions in Gothenburg AB, a GU Ventures-based platform company at the University of Gothenburg. K. Blennow has served as a consultant or at advisory boards for Alzheon, BioArctic, Biogen, Eli Lilly, Fujirebio Europe, IBL International, Merck, Novartis, Pfizer, and Roche Diagnostics, and is a cofounder of Brain Biomarker Solutions in Gothenburg AB, a GU Venture-based platform company at the University of Gothenburg. L. Forsgren reports no disclosures relevant to the manuscript. Go to Neurology.org/ $\mathrm{N}$ for full disclosures.

\section{Publication history}

Received by Neurology April 16, 2018. Accepted in final form August 15, 2018.

\section{References}

1. Macleod AD, Taylor KS, Counsell CE. Mortality in Parkinson's disease: a systematic review and meta-analysis. Mov Disord 2014;29:1615-1622.

2. de Lau LM, Breteler MM. Epidemiology of Parkinson's disease. Lancet Neurol 2006; 5:525-535.

3. Levy G, Tang MX, Louis ED, et al. The association of incident dementia with mortality in PD. Neurology 2002;59:1708-1713. 
4. De Pablo-Fernandez E, Tur C, Revesz T, Lees AJ, Holton JL, Warner TT. Association of autonomic dysfunction with disease progression and survival in Parkinson disease. JAMA Neurol 2017;74:970-976.

5. Litvan I, Goldman JG, Tröster AI, et al. Diagnostic criteria for mild cognitive impairment in Parkinson's disease: Movement Disorder Society Task Force guidelines. Mov Disord 2012;27:349-356.

6. Linder J, Stenlund H, Forsgren L. Incidence of Parkinson's disease and parkinsonism in northern Sweden: a population-based study. Mov Disord 2010;25:341-348.

7. Gibb WR, Lees AJ. The significance of the Lewy body in the diagnosis of idiopathic Parkinson's disease. Neuropathol Appl Neurobiol 1989;15:27-44.

8. Gilman S, Low PA, Quinn N, et al. Consensus statement on the diagnosis of multiple system atrophy. J Neurol Sci 1999;163:94-98.

9. Litvan I, Agid Y, Calne D, et al. Clinical research criteria for the diagnosis of progressive supranuclear palsy (Steele-Richardson-Olszewski syndrome): report of the NINDS-SPSP international workshop. Neurology 1996;47:1-9.

10. Domellöf ME, Elgh E, Forsgren L. The relation between cognition and motor dysfunction in drug-naive newly diagnosed patients with Parkinson's disease. Mov Disord 2011;26:2183-2189.

11. Jankovic J, McDermott M, Carter J, et al. Variable expression of Parkinson's disease: a base-line analysis of the DATATOP cohort. The Parkinson Study Group. Neurology 1990;40:1529-1534.

12. Doty RL, Marcus A, Lee WW. Development of the 12-item cross-cultural Smell Identification Test (CC-SIT). Laryngoscope 1996;106:353-356.

13. Podsiadlo D, Richardson S. The timed "Up \& Go": a test of basic functional mobility for frail elderly persons. J Am Geriatr Soc 1991;39:142-148.

14. Montgomery SA, Asberg M. A new depression scale designed to be sensitive to change. Br J Psychiatry 1979;134:382-389.

15. Blennow K, Wallin A, Agren H, et al. Tau protein in cerebrospinal fluid: a biochemical marker for axonal degeneration in Alzheimer disease? Mol Chem Neuropathol 1995; 26:231-245.

16. Vanmechelen E, Vanderstichele H, Davidsson P, et al. Quantification of tau phosphorylated at threonine 181 in human cerebrospinal fluid: a sandwich ELISA with a synthetic phosphopeptide for standardization. Neurosci Lett 2000;285:49-52.

17. Andreasen N, Hesse C, Davidsson P, et al. Cerebrospinal fluid $\beta$-amyloid (1-42) in Alzheimer disease: differences between early- and late-onset Alzheimer disease and stability during the course of disease. Arch Neurol 1999;56:673-680.

18. Mo SJ, Linder J, Forsgren L, et al. Pre- and postsynaptic dopamine SPECT in the early phase of idiopathic parkinsonism: a population-based study. Eur J Nucl Med Mol Imaging 2010;37:2154-2164.

19. Jakobson Mo S, Larsson A, Linder J, et al. ${ }^{123}$ I-FP-CIT and 123I-IBZM SPECT uptake in a prospective normal material analysed with two different semiquantitative image evaluation tools. Nucl Med Commun 2013;34:978-989.

20. Emre M, Aarsland D, Brown R, et al Clinical diagnostic criteria for dementia associated with Parkinson's disease. Mov Disord 2007;22:1689-1707; quiz 1837.

21. Yarnall AJ, Breen DP, Duncan GW, et al. Characterizing mild cognitive impairment in incident Parkinson disease: the ICICLE-PD study. Neurology 2014;82:308-316.

22. Hobson P, Meara J, Ishihara-Paul L. The estimated life expectancy in a community cohort of Parkinson's disease patients with and without dementia, compared with the UK population. J Neurol Neurosurg Psychiatry 2010;81:1093-1098.

23. Savica R, Grossardt BR, Bower JH, et al. Survival and causes of death among people with clinically diagnosed synucleinopathies with parkinsonism: a population-based study. JAMA Neurol 2017;74:839-846.

24. Nath U, Ben-Shlomo Y, Thomson RG, et al. Clinical features and natural history of progressive supranuclear palsy: a clinical cohort study. Neurology 2003;60:910-916.

25. Wenning GK, Geser F, Krismer F, et al. The natural history of multiple system atrophy: a prospective European cohort study. Lancet Neurol 2013;12:264-274.
26. Low PA, Reich SG, Jankovic J, et al. Natural history of multiple system atrophy in the USA: a prospective cohort study. Lancet Neurol 2015;14:710-719.

27. Ben-Shlomo Y, Marmot MG. Survival and cause of death in a cohort of patients with parkinsonism: possible clues to aetiology? J Neurol Neurosurg Psychiatry 1995;58: 293-299.

28. Roos RA, Jongen JC, van der Velde EA. Clinical course of patients with idiopathic Parkinson's disease. Mov Disord 1996;11:236-242.

29. Hoehn MM, Yahr MD. Parkinsonism onset, progression, and mortality. Neurology 1967;17:427-442.

30. Diamond SG, Markham CH, Hoehn MM, et al. An examination of male-female differences in progression and mortality of Parkinson's disease. Neurology 1990;40: 763-766.

31. Hughes AJ, Daniel SE, Kilford L, Lees AJ. Accuracy of clinical diagnosis of idiopathic Parkinson's disease: a clinico-pathological study of 100 cases. J Neurol Neurosurg Psychiatry 1992;55:181-184.

32. Driver JA, Kurth T, Buring JE, et al. Parkinson disease and risk of mortality: a prospective comorbidity-matched cohort study. Neurology 2008;70:1423-1430.

33. Marras C, McDermott MP, Rochon PA, et al. Survival in Parkinson disease: thirteenyear follow-up of the DATATOP cohort. Neurology 2005;64:87-93.

34. Forsaa EB, Larsen JP, Wentzel-Larsen T, Alves G. What predicts mortality in Parkinson disease? A prospective population-based long-term study. Neurology 2010;75: 1270-1276.

35. Otsuka M, Ichiya Y, Kuwabara Y, et al. Differences in the reduced $18 \mathrm{~F}-$ Dopa uptakes of the caudate and the putamen in Parkinson's disease: correlations with the three main symptoms. J Neurol Sci 1996;136:169-173.

36. Vanacore N, Spila-Alegiani S, Raschetti R, Meco G. Mortality cancer risk in parkinsonian patients: a population-based study. Neurology 1999;52:395-398.

37. Inzelberg R, Jankovic J. Are Parkinson disease patients protected from some but not all cancers? Neurology 2007;69:1542-1550.

38. Ritz B, Ascherio A, Checkoway H, et al. Pooled analysis of tobacco use and risk of Parkinson disease. Arch Neurol 2007;64:990-997.

39. Noyce AJ, Bestwick JP, Silveira-Moriyama L, et al. Meta-analysis of early nonmotor features and risk factors for Parkinson disease. Ann Neurol 2012;72:893-901.

40. Willis AW, Schootman M, Kung N, et al. Predictors of survival in patients with Parkinson disease. Arch Neurol 2012;69:601-607.

41. Marié RM, Barré L, Dupuy B, et al. Relationships between striatal dopamine denervation and frontal executive tests in Parkinson's disease. Neurosci Lett 1999;260:77-80.

42. Ekman U, Eriksson J, Forsgren L, et al. Functional brain activity and presynaptic dopamine uptake in patients with Parkinson's disease and mild cognitive impairment: a cross-sectional study. Lancet Neurol 2012;11:679-687.

43. Irwin DJ, Grossman M, Weintraub D, et al. Neuropathological and genetic correlates of survival and dementia onset in synucleinopathies: a retrospective analysis. Lancet Neurol 2017;16:55-65.

44. Baufeld C, O'Loughlin E, Calcagno N, et al. Differential contribution of microglia and monocytes in neurodegenerative diseases. J Neural Transm 2018;125:809-826.

45. Grozdanov V, Bliederhaeuser C, Ruf WP, et al. Inflammatory dysregulation of blood monocytes in Parkinson's disease patients. Acta Neuropathol 2014;128:651-663.

46. Chen $\mathrm{H}$, Zhang SM, Hernán MA, et al. Nonsteroidal anti-inflammatory drugs and the risk of Parkinson disease. Arch Neurol 2003;60:1059-1064.

47. Chen H, Jacobs E, Schwarzschild MA, et al. Nonsteroidal antiinflammatory drug use and the risk for Parkinson's disease. Ann Neurol 2005;58:963-967.

48. Adler CH, Beach TG, Hentz JG, et al. Low clinical diagnostic accuracy of early vs advanced Parkinson disease: clinicopathologic study. Neurology 2014;83:406-412.

49. Hughes AJ, Daniel SE, Ben-Shlomo Y, Lees AJ. The accuracy of diagnosis of parkinsonian syndromes in a specialist movement disorder service. Brain 2002;125: $861-870$. 


\section{Neurology}

\section{Early predictors of mortality in parkinsonism and Parkinson disease: A population-based study}

David Bäckström, Gabriel Granåsen, Magdalena Eriksson Domellöf, et al. Neurology 2018;91;e2045-e2056 Published Online before print October 31, 2018

DOI 10.1212/WNL.0000000000006576

This information is current as of October 31, 2018

Neurology ${ }^{\circledR}$ is the official journal of the American Academy of Neurology. Published continuously since 1951, it is now a weekly with 48 issues per year. Copyright Copyright (C) 2018 The Author(s). Published by Wolters Kluwer Health, Inc. on behalf of the American Academy of Neurology.. All rights reserved. Print ISSN: 0028-3878. Online ISSN: 1526-632X.

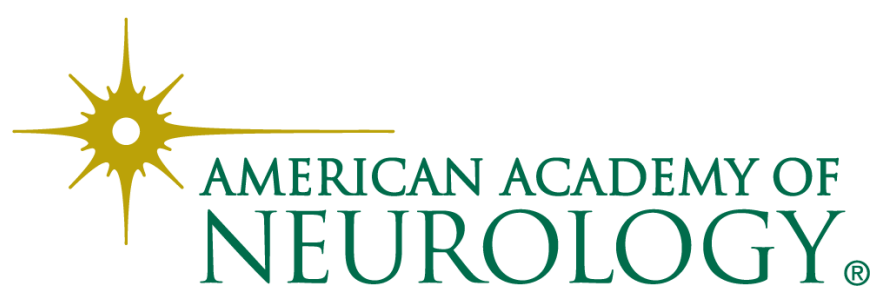




\section{Updated Information \& Services}

\section{Supplementary Material}

References

Citations

Subspecialty Collections

\section{Permissions \& Licensing}

Reprints including high resolution figures, can be found at: http://n.neurology.org/content/91/22/e2045.full

Supplementary material can be found at: http://n.neurology.org/content/suppl/2020/03/04/WNL.0000000000006 576.DC1

This article cites 49 articles, 17 of which you can access for free at: http://n.neurology.org/content/91/22/e2045.full\#ref-list-1

This article has been cited by 6 HighWire-hosted articles: http://n.neurology.org/content/91/22/e2045.full\#\#otherarticles

This article, along with others on similar topics, appears in the following collection(s):

\section{All Imaging}

http://n.neurology.org/cgi/collection/all_imaging

Multiple system atrophy

http://n.neurology.org/cgi/collection/multiple_system_atrophy

Natural history studies (prognosis)

http://n.neurology.org/cgi/collection/natural_history_studies_prognosis

Parkinson's disease/Parkinsonism

http://n.neurology.org/cgi/collection/parkinsons_disease_parkinsonism Progressive supranuclear palsy

http://n.neurology.org/cgi/collection/progressive_supranuclear_palsy

Information about reproducing this article in parts (figures,tables) or in its entirety can be found online at:

http://www.neurology.org/about/about_the_journal\#permissions

Information about ordering reprints can be found online:

http://n.neurology.org/subscribers/advertise

Neurology ${ }^{\circledR}$ is the official journal of the American Academy of Neurology. Published continuously since 1951, it is now a weekly with 48 issues per year. Copyright Copyright ( 2018 The Author(s). Published by Wolters Kluwer Health, Inc. on behalf of the American Academy of Neurology.. All rights reserved. Print ISSN: 0028-3878. Online ISSN: 1526-632X.

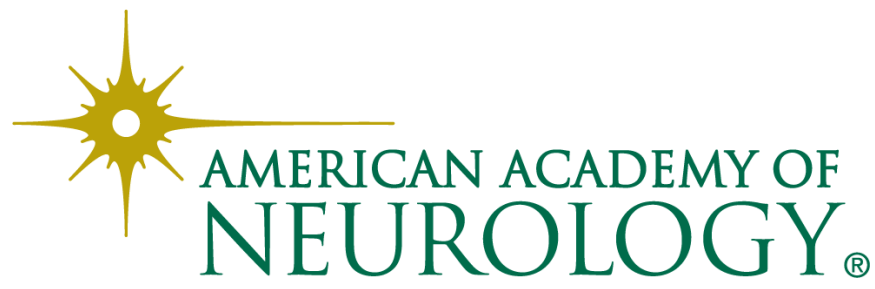

